\title{
Benefits of implementing IT tools and procedures for the estimation and measurement uncertainty in testing laboratories
}

\author{
Maria-Cristina Dijmarescu \\ University Politehnica of Bucharest, Quality Engineering and Industrial Technologies \\ Department, Splaiul Independentei 313, 060042, Bucharest, Romania \\ maria.dijmarescu@upb.ro
}

\begin{abstract}
Destructive and non-destructive testing of materials present a rapid expansion given by the increase in market demand caused by the desire to obtain an increasingly better quality of products. The continuous increase in quality demands leads directly to the need to implement and modernize the techniques, methods, and equipment used for quality control. Consequently, the need for product testing services has a rapid growth. This paper presents the strength and weaknesses of implementing IT tools for the estimation of the measurement uncertainty in testing laboratories and the impact of these tools on the economic part.
\end{abstract}

Keywords. uncertainty measurement, testing, IT tools, procedures.

\section{Introduction}

In order to carry out quality testing for the customers, laboratories must first obtain an accreditation certificate. The accreditation represents third-party attestation, which represents the confirmation of the very fact that a body is competent to perform specific conformity assessment tasks. It helps to extend the competitiveness of products and services within the context of market globalization.

In Romania, the certificate is given by the national accreditation body - RENAR.

According to the ISO/IEC 17025 standard, testing laboratories must have implemented the following:

- When assessing measurement uncertainty, all significant contributions, including those resulting from sampling, should be considered using appropriate methods of analysis.

- A laboratory shall evaluate the measurement uncertainty for all calibrations including of its own equipment.

- To evaluate the measurement uncertainty it's mandatory in a laboratory performing testing. Within the cases when the measurement uncertainty will be based on the theoretical principles or on the experience of the performance of the measurement method used the rigorous evaluation will be precluded.

Uncertainty of measurement generally includes several components. Some of these components are evaluated based on distribution statistics and measurement results and are characterized as deviation standards. The other components are analyzed as probability deviations and are based on experience and information [1-4]. 
„The uncertainty of the result of a measurement reflects the impossibility of accurately knowing the measurement value.

The result of a measurement, after correction for the identified systemic effects, remains only an estimate of the measurement value due to the uncertainty resulting from the random effects and the imperfect correction of the result for systematic effects" [5].

In estimating the uncertainty of the outcome of a measurement, all components, including those derived from systematic effects, such as components associated with corrections and references values, should be considered, contributing to the dissemination of the results $[3,5]$.

Measurement uncertainty is a common concept. This gives us a certain degree of confidence in the result obtained from a measurement. However, its estimation is a very complex process, requiring knowledge of mathematics, first, but also a thorough knowledge of the method by which a certain type of measurement is made [6-7].

The instruments, calibration blocks and standards used to perform the measurements are accompanied by their own measurement uncertainty, to ease the operator's work [8].

Scientists have created and developed tools to optimize their work in order to reduce the time lost by making the mathematical calculations necessary to estimate uncertainty of measurement, regardless of the type of measurement [6-8].

\section{Measurement uncertainty estimation}

The concept of „uncertainty" it is relatively new and represent an associate parameter of the measurement result which characterizes the dispersion of the values that can reasonably be attributed to the measurement $[1-2,5]$.

The result of the measurement uncertainty reflects the lack of knowledge of the measurand value. Taking into account the uncertainty arising from random effects and from imperfect correction of the result for systematic effects after the correction for recognized systematic values the measurement result it is still only a value estimate of the of the measurand because of the $[2-3,5]$.

For estimating the value of the measurement uncertainty for a particular method the input quantities that characterizes it and the factors which can influence the value of the uncertainty must be established [1, 9-10].

The purpose of the measurement is to determine the value of a magnitude of interest, such as the Rockwell value of the hardness of a metal sample, the stress test, the length of a sample at $20^{\circ} \mathrm{C}$, etc. [5].

Each measurement has an associated uncertainty. The measuring devices, calibration blocks are not perfect too, environmental conditions, processes, procedures, and people are imperfect and variable $[1,5,11]$.

In order to compare two measurements of uncertainty, they must have the same reference. The method by which the measurement uncertainty is calculated depends on the nature of the test and may be simple or complex in order to meet the imposed requirements. Measurement of uncertainty is not only important for calibration, but also for any test involving measurements $[1,3,11]$.

As a result of the study of the scientific literature, a large part of the norms related to the estimation of measurement uncertainty are based on the reference "GUM - Guide to the Expression of Uncertainty in Measurement" of 1995.

Currently, the International Organization for Standardization has developed and continues to develop a set of norms for estimating uncertainty of measurement.

In Romania, it is used the "SR ENV 13005: 2003 Guide to expression of uncertain measurement" standard, which is also based on GUM: 1995 normative principles.

According to the JCGM 100:2008 standard, to evaluate and express the measurement uncertainty, must be respected the next procedure: 
- the mathematical expression of the relationship between the measurand, $\mathrm{Y}$, and the input quantities, $\mathrm{Xi}$, on which the function depends, must also include the correction factors required for each size.

- determining the estimated value; xi, for each input quantity.

- evaluation of the standard uncertainty, $u(x i)$, for each estimated value.

- evaluation of covariates associated with the input's quantities estimate.

- determining combined standard uncertainty, $\mathrm{u}_{\mathrm{c}}(\mathrm{y})$.

- determining expanded uncertainty, U.

- reporting the result.

When reporting the result of a measurement, an indication of its quality should also be provided so that those who will use it later can assess its credibility [2, 12-13].

In practice, the uncertainty possible sources can be represented by [2, 5, 10-11]:

- incomplete definition of the measurand.

- imperfect definition of the measurand.

- nonrepresentative sampling of the defined measurand.

- lack of knowledge of the environmental conditions or improper conditions.

- personal bias in reading analogue instruments.

- finite instrument resolutions od discrimination threshold.

- inexact values of measurement standards and references materials.

- inexact values of constants and other parameters obtained from external sources.

- approximations and assumptions incorporated in the measurement method and procedure.

- variations in repeated observations of the measurand under identical conditions.

In order to establish the necessary mathematical steps and relationships, it is necessary to identify and characterize the main factors that influence the outcome of a measurement and the steps to be followed $[6,12]$.

In Figure 1, the basic stages and their sequence are presented - the steps taken to determine the uncertainty of measurement.
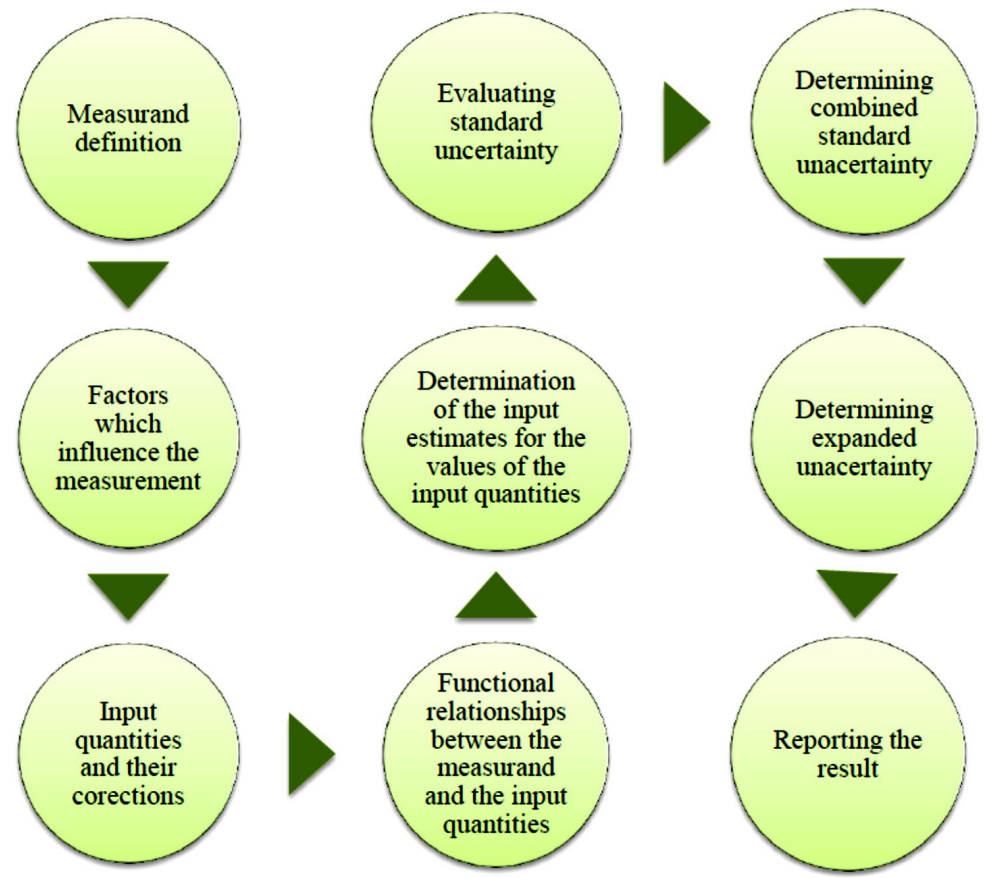

Figure 1. Steps for the measurement uncertainty estimation 2.1. Factors which influence the measurement uncertainty 
To determine the mathematical model, four groups of factors were considered, namely: the analysed sample, the used equipment, the working environment and the operator.

Each group has highlighted the fundamental factors that can influence the outcome of a measurement in testing laboratories, as shown in Figure 2.

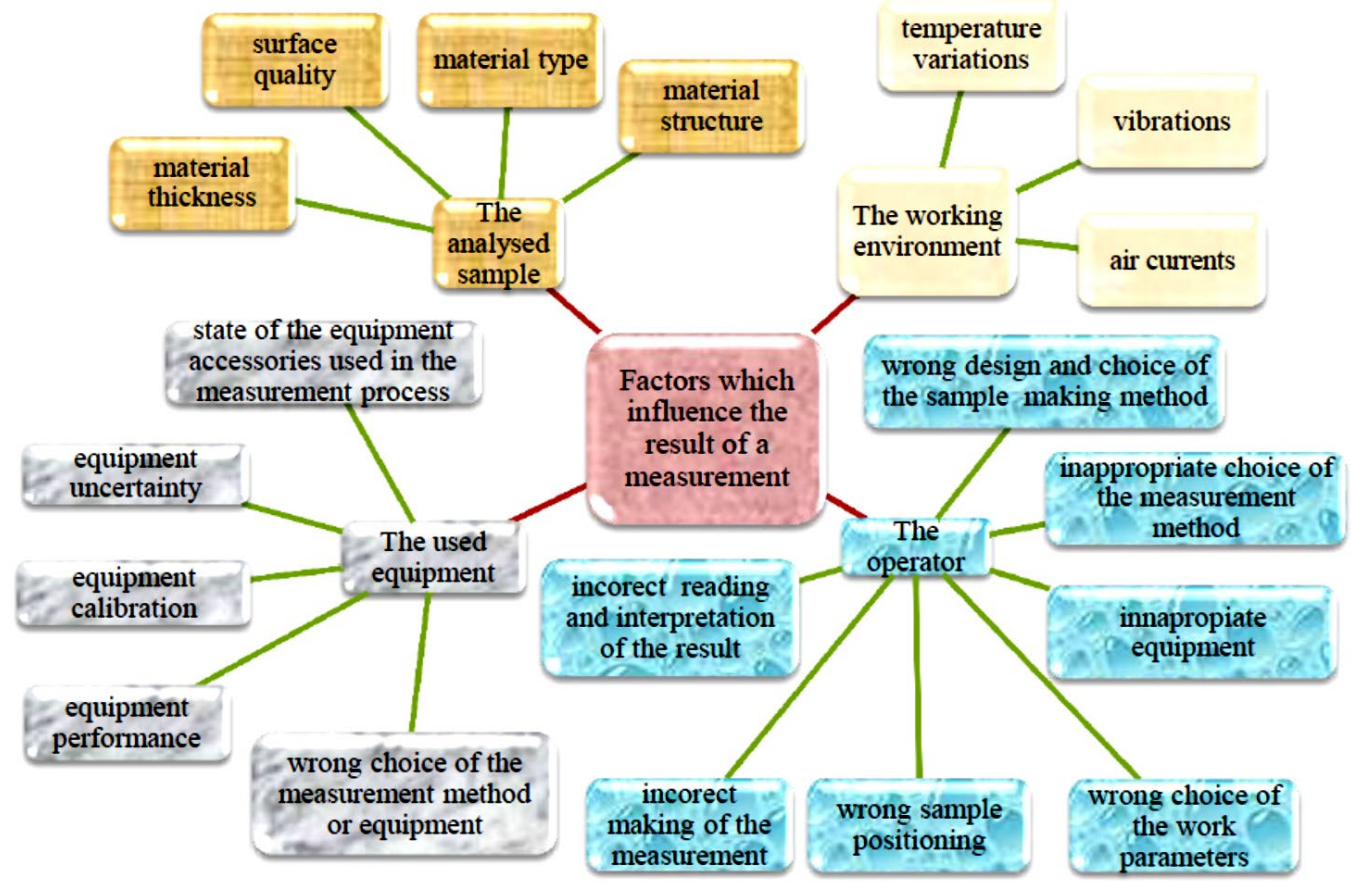

Figure 2. Factors which influence the result of a measurement

\section{The benefits of implementing IT tools}

In order to create a testing laboratory with the main activity consisting in providing complete services in the field of destructive and non-destructive testing of materials and products in the industrial field the SWOT method was used.

The strategic vision of the laboratory is targeting of small, medium and large companies in the field of production and construction of machines within the EEA (European Economic Area), in the naval field and in the fields of pressure vessels. In figure 3 are presented the main testing methods provided by the laboratory.

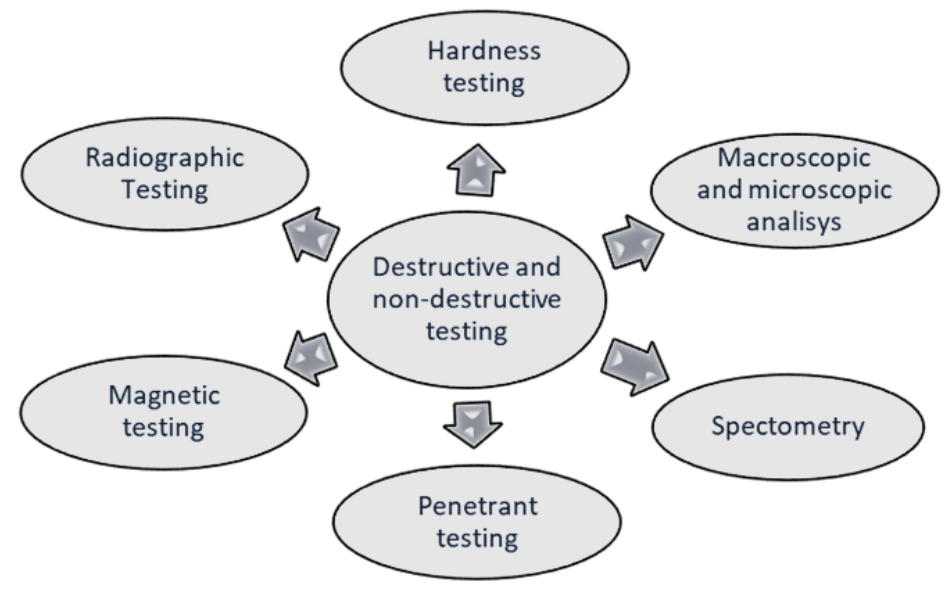

Figure 3. Main testing methods provided 
Considering the requirements of SR EN ISO / CEI 17025: 2018 standard referring to the fact that testing laboratories must have and apply procedures to estimate the uncertainty measurements, the laboratory will implement IT tools developed for calculate this coefficient and specific procedures for all the testing methods provided. The computer tools are developed and adapted to the conditions present in the laboratory.

The laboratory will adopt the differentiation strategy by implementing and developing innovative software to reduce time and costs. The laboratory aims to create a special advantage, based on a unique factor that is felt throughout the field and appreciated by a large part of customers.

This factor being represented by the accompaniment of the result obtained after the measurement analysis by the uncertainty, in a short amount of time. Thus, the laboratory will focus on achieving superior performance, which ensures its leading position in the sector through the technology used.

Critical success factors identified for the business field are:

- Diversification of the existing control methods within the laboratory.

- Providing a result with a satisfactory degree of confidence.

- Implementation of state-of-the-art software for interpreting the results.

- Adaptability and market resilience.

For a better overview of the company a SWOT analysis was applied, presented in figure 4.

- Ability to adopt a competitive price position

-A detailed analysis of all the factors influencing the measurement uncertainty and specific procedures for reducing it.

-The use of state-of-the-art software, but also software created to reduce the calculation time of the measurement uncertainty.

- Short duration for calculation of measurement uncertainty

- Providing results accompanied by measurement uncertainty in a short amount of time.

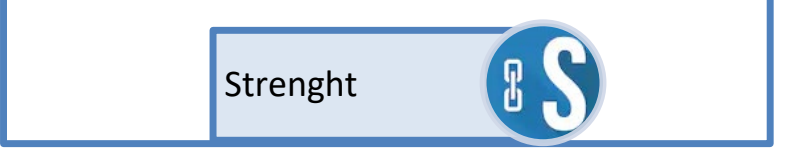

- Competitive and normative pressures exerted on industrial factors.

- Development and Implementation of new technologies with a high degree of performance.

-Economic growth given by the reduced time used to calculate the uncertainty.

- Partnerships with major industrial actors and / or commercial partners in order to perform tests under accreditation conditions

\section{Opportunities}

- Lack of human capital needed to tackle largescale projects.

- High costs of purchasing equipment.

-Re-accreditation costs.

- High costs for operator training.

- Lack of computer knwolege of the operator
- Substantial indirect costs related to the acquisition of new technological means

- Development of internal capacities for the implementation of new technologies.

- Hiring or training level three operators for the new methods implemented

-The rapid evolution of the market, as well as the need to examine new materials (additive materials)

Threats

Figure 4. SWOT analysis 
A mathematical model is provided by the JCGM 100: 2008 standard that can be applied to a measurement technique.

Depending on the measurement technique and the type of input quantities that characterizes the measurand, changes to the pattern may occur, they refer to new formulas where the value of a particular input is obtained.

First of all, the estimation of the measurement uncertainty is a very complex process, which requires knowledge of mathematics, but also a thorough knowledge of the method by which a certain type of measurement is performed.

In order to gain economic benefits, the following improvement measures can be applied in the testing laboratory:

- The making of procedures for the identification of the influence factors and for the estimation of measurement uncertainty for each method in order to reduce the workload of the operator.

- The implementation of IT tools for the estimation of the measurement uncertainty for each method, in order to reduce the time lost with the mathematical calculations.

Normally a procedure of the measurement uncertainty estimation provides only the mathematical model for that method and the laboratory environment conditions, if the identification of the influence factors is added, the work of the operator will be reduced, and time can be gained. Each disturbing factor influences in a particular way and by a specific feature the measurement result. In estimating uncertainty of measurement, must be taken into account the importance of each factor and the logical order and the extent to which it influences the measurement result.

Also, if the model is already particularized for a certain method it will be easier to identify the input quantities to estimate the covariates associated with the input's quantities estimate.

The implementation of IT tools for the estimation of the measurement uncertainty in the laboratory reduce the time spent by the operators to calculate it and to search for the test calibre measurement.

The software can contain different function that retain the information needed for a certain measurement and the operator must input only the measurements without performing the test on the calibre sample.

\section{Conclusions}

This paper presented and discussed the importance of the uncertainty measurement for a test method and the importance of creating tools for estimating the measurement uncertainty in order to obtain a quality result in a short amount of time.

In the first part of the second section of the paper the main standards used to calculate the uncertainty and the steps needed to estimate the measurement uncertainty are presented. In the last part of this section the main factors that influence the value of the uncertainty in destructive and nondestructive testing were identified.

In the third part of the paper for the laboratory takes as a test subject are presented the strategic vision, the main testing methods provided, the strategy implemented and the critical factors. Followed by the SWOT analysis and the highlighting a part of the benefits of the implementations of procedures and IT tools for the estimation of measurement uncertainty.

Using the SWOT analysis were identified the strengths, weaknesses, opportunities and threats for a laboratory which has IT tools implemented.

\section{Acknowledgment}

This work has been funded by the European Social Fund from the Sectoral Operational Programme Human Capital 2014-2020, through the Financial Agreement with the title "Scholarships for entrepreneurial education among doctoral students and postdoctoral researchers (Be Antreprenor!)", Contract no. 51680/09.07.2019 - SMIS code: 124539. 


\section{References}

[1] EURAMET/cg-16/v.01 2007: Guidelines on the Estimation of Uncertainty in Hardness Measurements.

[2] G104/ 2014: Guide for Estimation of Measurement Uncertainty In Testing, American Association for Laboratory Accreditation.

[3] SR ENV 13005: 2003: Ghid pentru exprimarea incertitudinii de măsurare, inlocuieşte SR 13434: 1999 Ghidul pentru Exprimarea Incertitudinii de Măsurare (GUM).

[4] ISO/IEC17025:2017: General requirements for the competence of testing and calibration laboratories.

[5] JCGM100:2008: Evaluation of measurement data - Guide to the expression of uncertainty in measurement (Joint Committee for Guidesin Metrology).

[6] J. M. BALDWIN, K. D. SUMMERHAYS, D. A. CAMPBELL, R. P. HENKE: Application of Simulation Software to Coordinate Measurement Uncertainty Evaluations, $J$. of Measurement Science, ISSN: 2381-0580, 2 (4), 40-52, (2007).

[7] J. BEAMAN, E. MORSE: Experimental evaluation of software estimates of task specific measurement uncertainty for CMMs, Precision Engineering, ISSN 0141-6359, 34 (1), 2833, (2010).

[8] T. NÄYKKI, A. VIRTANEN, I. LEITO: Software support for the Nordtest method of measurement uncertainty evaluation, Accreditation and Quality Assurance, ISSN 1432-0517, 17, 603-612, (2012).

[9] EA-4/16: 2003: Exprimarea incertitudinii în încercările cantitative, (EA Guide-lines on the Expression of Uncertainty in Quantitative Testing).

[10] ILAC G17: 2002: Introducing the Concept of Uncertainity of measurement in testing in Association with the application of standard ISO/IEC 17025.

[11] JCGM 101:2008: Evaluation of measurement data-Supplement 1 to the "Guide to the expression of uncertainty in measurement" - Propagation of distributions using a Monte Carlo method (ISO/IEC Guide 98-3-1.

[12] M.C. DIJMĂRESCU, M.R. DIJMĂRESCU: Estimation of Vickers hardness uncertainty for a heterogeneous welded joint (S235JR+AR and X2CrNiMo17-12-2), IOP Conf. Series: Materials Science and Engineering, ISSN: 1757-8981, 227, 012025, (2017).

[13] M.C. DIJMĂRESCU, M.R. DIJMĂRESCU: Design and Development of a Software for the Estimation of the Vickers Hardness Measurement Uncertainty, IOP Conf. Series: Materials Science and Engineering, ISSN: 1757-8981, 916, 012025, (2020). 